\title{
El Neuromarketing en el Ecuador: ESTUDIO COMPARATIVO CON EL MARKETING TRADICIONAL Y LA CONSTRUCCIÓN de UN OCÉANO AZUL
}

\author{
Neuromarketing in ECUAdOR: Comparative STUdy \\ WITH TRADITIONAL MARKETING AND THE CONSTRUCTION OF A BLUE OCEAN
}

Mg. Gabriela Pástor

Universidad Internacional SEK - Ecuador

Ph.D. Fabrizio Jácome

Universidad Tecnológica Equinoccial - Ecuador

Ph. D. Diego Donoso V.

Universidad Tecnológica Israel - Ecuador

ddonoso@uisrael.edu.ec

\section{Resumen}

Fecha de recepción: 04/05/2016

Fecha de aceptación: 07/06/2016

El propósito de esta publicación está basado en la necesidad que tenemos de dejar a un lado la competencia destructiva que existe hoy en día entre las empresas, para poder tener éxito en el futuro, es necesario ampliar los horizontes del mercado y generar valor a través de la innovación. "Los océanos rojos representan todas las industrias que existen en la actualidad, mientras que los océanos azules simbolizan las ideas de negocio hoy por hoy desconocidas" (Chan W. y Mauborgne R. 2005. p.1) 
A través de la estrategia de océano azul demostraremos la importancia de manejar nuevas metodologías estratégicas en nuestras empresas entrando en un mundo desconocido para muchos, como es el ámbito de la subjetividad humana en donde nosotros aplicamos procesos de orden emotivo es decir, cautivamos al consumidor a través de los distintos estímulos de publicidad, marca, promoción, entre otras, para que se sienta atraído por lo que le estamos ofreciendo,

Palabras clave:Neuromarketing, Marketing, Océano Azul, Mercado

\section{Abstract}

The purpose of this publication is based on the need to put aside the destructive competition that exists today between companies, in order to be successful in the future, it is necessary to broaden the horizons of the market and generate value through innovation. "The red oceans represent all the industries that exist today, while the blue oceans symbolize today's unknown business ideas" (Chan W. and Mauborgne R. 2005. p.1)

Through the blue ocean strategy we will demonstrate the importance of managing new strategic methodologies in our companies entering a world unknown to many, as it is the field of human subjectivity where we apply processes of emotional order ie captivate the consumer to Through the various stimuli of advertising, brand, promotion, among others, so that you are attracted by what we are offering.

Keywords: Neuromarketing, Marketing, Blue Ocean, Market 


\section{Introducción}

En la actualidad las empresas se ven amenazadas por la cada vez más creciente competencia que hay en el mercado ecuatoriano. El cliente tiene una gran cantidad de opciones al momento de elegir un producto. Pero; ¿qué es lo que verdaderamente pasa en su cerebro para que lo lleve a tomar una decisión?, ó ¿qué influye para que este decida sobre una marca u otra?

Para responder estas preguntas los científicos han indagado el cerebro humano durante años, es por esto que la década de los noventa fue conocida como la "Década del cerebro" debido a que en solo este tiempo el avance en el conocimiento del cerebro ha sido mayor que en todos los miles de años anteriores de la historia de la humanidad, y los avances de las Neurociencias han traído aplicaciones en campos como el Neuromarketing, Neuromanagement, y la venta Neurorrelacional (Biurrun C. 2011 párr. 4).

Nos centraremos en el neuromarketing, un nuevo campo del marketing que trae consigo una forma novedosa de investigar la respuesta cerebral a los estímulos: publicitarios, de marca, posicionamiento, precios, comunicaciones, canales, etc. El neuromarketing se define como el estudio del funcionamiento del cerebro en las decisiones de compra de un producto o servicio. Según Sergio Monge (2009. párr. 2) "nació de la fusión de las neurociencias y las técnicas de investigación del marketing tradicional". Estas nuevas estrategias del neuromarketing nos dan la herramienta y nos enseñan cómo estructurar y definir nuestros mensajes de tal manera que impactemos con ellos en la máquina de tomar decisiones ${ }^{1}$ de nuestros interlocutores.

\section{Planteamiento}

El presente trabajo tiene como objetivo plantear un nuevo modelo de promocionar nuestros productos, basado en la estrategia de océanos azules. Esta teoría nos dice que la clave de éxito de toda empresa es crear sus propias formas de negocio, alejándose así de su competencia y creando estrategias completamente nuevas y novedosas para lograr un océano azul.

1 Máquina de tomar decisiones: el cerebro humano y sus reacciones ante estímulos de mensajes y publicidad. 
En el marketing tradicional nosotros nos hacemos preguntas como estas: ¿qué debemos poner en un comercial para que este tenga más impacto en la audiencia?, ¿qué estrategias de precios usar?, ¿qué hacer para que nuestra empresa sea competitiva ante otras fuerzas de ventas?, ¿qué medios de comunicación usar y cuantas veces debe salir nuestra publicidad? ¿Cómo logro fidelidad de nuestra marca?, etc. Y creemos tener la respuesta haciendo encuestas de investigación a nuestros clientes, preguntándoles: ¿te gusta este producto? ¿por qué?

La respuesta puede estar influenciada por las vías $\operatorname{cognitivas}^{2}$ y por la imperfección del lenguaje ${ }^{3}$, ya que el $85 \%$ de la conducta se guía por el subconsciente. (Fundación Telefónica, 2011). Al realizar este tipo de cuestionarios, las respuestas repercutirán en el resultado final de nuestra investigación de mercado, las respuestas puede que no reflejen la realidad de los hábitos, preferencias y percepciones de nuestros consumidores.

El neuromarketing nos permite realizar una investigación de mercados especializada, para obtener datos que nos muestran, cómo reacciona el consumidor ante diferentes estímulos a los que es expuesto.

Por consiguiente el neuromarketing indaga cuáles zonas del cerebro están involucradas en el comportamiento del cliente, ya sea cuando compramos un producto, cuando elegimos una marca, o simplemente, cuando recibimos e interpretamos los mensajes que nos hacen llegar las marcas. "Trata de buscar el botón de compra que, parece ser, todos tenemos en el cerebro" (Olamendi, 2001).

El neuromarketing es desconocido por las empresas ecuatorianas, debido a que no poseen los conocimientos para implementarlo limitándose únicamente a manejar el marketing tradicional como hasta ahora todas las empresas lo han hecho.

\section{Sustentación}

A continuación se muestra en la Tabla $\mathrm{N}^{\circ} 1$, las variables estratégicas actuales que conforman el océano rojo, son todas las empresas que siguen el mismo esquema y se manejan bajo el uso de las mismas estrategias de marketing sin lograr diferenciación alguna.

El proceso de descubrir y crear un océano azul no consiste en intentar predecir las tendencias de una industria o sector a través de un ejercicio meramente subjetivo. Tampoco consiste en implementar las nuevas ideas que surgen en la mente de los gestores utilizando el método de ensayo y error.

2 Vías Cognitivas: Tendencia de los seres humanos a sobrevalorar la disposición subjetiva de una persona debido a las circunstancias ambientales.

3 Imperfección del Lenguaje: No siempre se expresa lo que objetivamente pensamos 
Tabla $N^{\circ} 1$ : Variables Estratégicas

\begin{tabular}{|c|c|}
\hline V1 & Publicidad excesiva \\
\hline V2 & Escoger un grupo objetivo \\
\hline V3 & Promoción \\
\hline V4 & Estudio de mercado \\
\hline V5 & Producción de piezas publicitarias \\
\hline V6 & Medios \\
\hline V7 & Posicionamiento \\
\hline
\end{tabular}

Fuente: Elaborado por G. Pástor.

"El primer principio para la creación de una estrategia de estas características es establecer un proceso estructurado que logre ampliar los límites del mercado tal y como se concibe hoy en día". (Chan W. y Mauborgne R. 2005.p.2)

La mayoría de las empresas ecuatorianas se encuentran actualmente en océanos rojos, manejan el marketing de manera similar, apenas con ligeros cambios en el diseño de productos, mostrando demasiada publicidad para crear posicionamiento, branding[4], comunicación, distribución, en fin tareas que son necesarias pero al complementarlas con nuevas estrategias podrían ser sumamente exitosas.

El neuromarketing nos da la oportunidad de crear un océano azul en el mercado ecuatoriano, para poder conocer lo que nuestros clientes quieren y ofrecerles un producto, que va a estar acorde con sus gustos y preferencias.

Por consiguiente, lo primero que las empresas ecuatorianas deben hacer es trabajar en su lienzo estratégico, realizando una Matriz RECA - RICE en donde se tendrá claro qué es lo que se tiene que eliminar, reducir, aumentar y crear.

El objetivo principal de toda empresa es obtener altos márgenes de ganancia en relación a sus ventas, pero ¿qué estamos haciendo para conseguir esto?, en la matriz de Rice claramente se observa, que tenemos que disminuir la publicidad excesiva, ya que con las nuevas estrategias mencionadas no es necesario promocionar tanto nuestros productos para ganar posicionamiento. 
Tabla No2: Matriz RICE

\begin{tabular}{|c|c|}
\hline \multicolumn{1}{|c|}{ Eliminar } & \multicolumn{1}{c|}{ Reducir } \\
\hline - Publicidad excesiva & $\begin{array}{l}\text { Escoger un grupo objetivo } \\
\text { Promoción }\end{array}$ \\
\hline \multicolumn{1}{|c|}{ Aumentar } & \\
\hline - Estudio de mercado & Crear \\
\hline - Producción de piezas publicitarias & - Respuesta cerebral a estímulos publicitarios \\
\hline $\begin{array}{l}\text { Medios } \\
\text { Posicionamiento }\end{array}$ & $\begin{array}{l}\text { Nuevas estratégias a través de la introducción } \\
\text { al parámetro de la subjetividad humana. }\end{array}$ \\
\hline
\end{tabular}

Fuente: Elaborado por G. Pástor.

Esto se debe a que el neuromarketing nos permite obtener datos para analizar la conducta del consumidor y así poder conocer sus deseos y saber qué es lo que les motiva a comprar un producto o a elegir un servicio independientemente de la marca que sea, del tamaño de organización o del tipo de consumidor que este sea.

Gráfico Nº1: Océano Azul entre El Marketing Tradicional y el Neuromarketing.

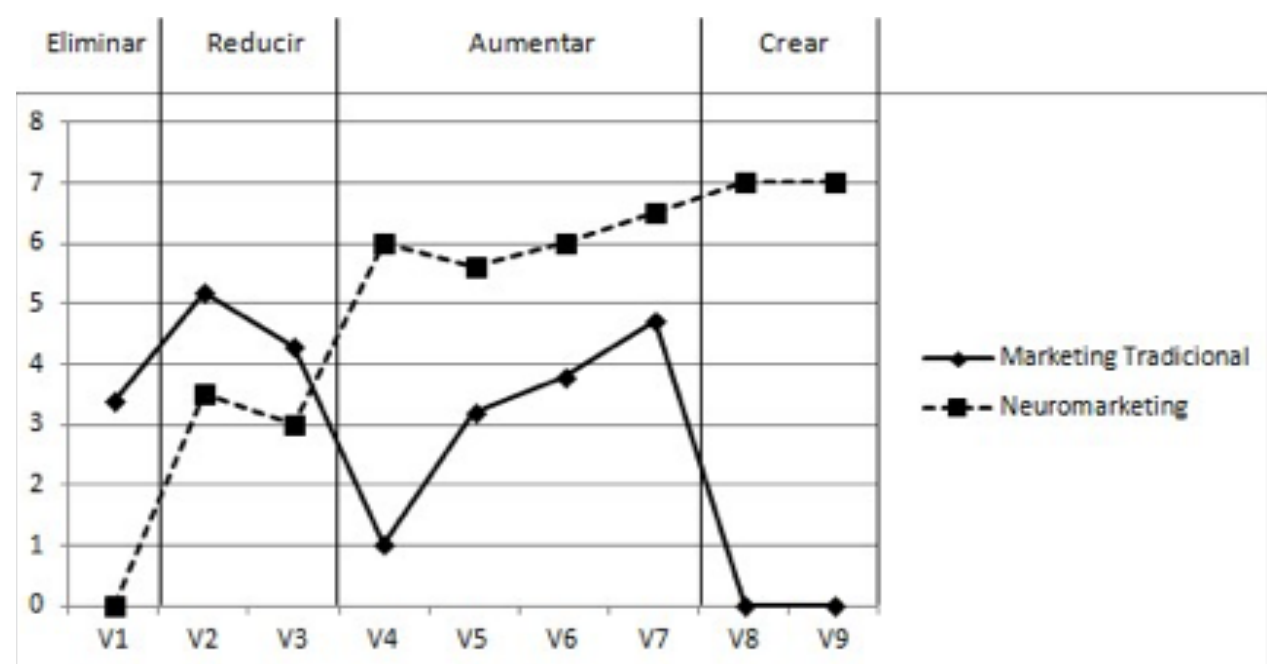

Fuente: Elaborado por G. Pástor. 
Para ilustrar de mejor manera como una empresa puede diferenciarse de las demás basándose en esta estrategia, es necesario que observemos gráficamente como se puede pasar de una economía que se encuentra en el océano rojo a una en particular que se encuentra en el océano azul.

Las variables están calificadas en una escala del 1 al 8 haciendo una comparación entre las empresas ecuatorianas que manejan el Marketing Tradicional y la propuesta de crear una nueva estrategia aplicando las técnicas de neuromarketing.

El estudio de la respuesta cerebral que nuestros posibles consumidores van a tener a los estímulos publicitarios que nosotros les mostraremos a través de la publicidad para saber si el producto que nosotros vamos a crear, va a ser de agrado para el consumidor.

Tabla N³: Variables estratégicas del Gráfico $\mathrm{N}^{\circ} 1$

\begin{tabular}{|c|c|}
\hline V1 & Publicidad excesiva \\
\hline V2 & Escoger un grupo objetivo \\
\hline V3 & Promoción \\
\hline V4 & Estudio de mercado \\
\hline V5 & Producción de piezas publicitarias \\
\hline V6 & Podios \\
\hline V7 & Posionamiento \\
\hline V8 & Respuesta cerebral a estímulos publicitarios \\
\hline V9 & Nuevas estrategias a través de la introducción al paráme- \\
\hline
\end{tabular}

Fuente: Elaborado por G. Pástor.

Esto es importante porque tendremos la certeza de que el estudio que estamos haciendo tendrá una respuesta confiable en cuanto a la investigación de mercados, conociendo realmente lo que las personas piensan sin la interferencia de emociones que puedan variar la respuesta final. 


\section{Conclusiones}

Es importante saber que hoy en día con tanta empresa competidora se torna muy difícil diferenciarnos al promocionar nuestros productos, pero hay que tener en cuenta que existen nuevas metodologías que nos pueden ayudar a ser diferentes ante los ojos de nuestro cliente, y a incrementar nuestras ventas creando un Océano Azul.

Tenemos que aprovechar la oportunidad de crear algo nuevo y diferente ya que en la teoría clásica los estudios se basan únicamente en los procesos de orden racional, para la maximización de beneficios y minimización de costos, pero resulta que esto no es suficiente para entender la complejidad humana y la complejidad de los mercados.

Es necesario mirar que en este proceso no solamente se pueden ver variables cuantificables, sino que tenemos que introducirnos al parámetro de la subjetividad humana[5] y especialmente a lo que tiene que ver con el neuromarketing.

Finalmente tengamos en cuenta que el neuromarketing acompañado del marketing tradicional nos puede llevar a crear productos, servicios y campañas de publicidad, completamente nuevas y exitosas conociendo los impactos que estos tendrían en los niveles inconscientes de nuestro consumidor y creando un nuevo Océano Azul. 


\section{Bibliografía}

Biurrun, C. (2011). Neuromarketing y seguro, el blog de Carlos Biurrun. Consulta el 25 de noviembre 2011, de http://issuu.com/cejotabe/docs/eletter_bdecb

Braidot, N. (2009). Neuromarketing, ¿Por qué tus clientes se acuestan con otro si dicen que les gustas tú?. España. Gestión 2000.

Braidot, N. (2006). Venta Inteligente, El método de Venta Neurorrelacional. España. Puerto Norte Sur.

Carletti, E. (2010). Neuromarketing: Para vender más, estudian el cerebro de la gente. Consulta 13 diciembre 2011, de http://axxon. com.ar/noticias/2010/03/neuromarketing-para-vender-masestudian-el-cerebro-de-la-gente/.

Chan W., y Mauborgne, R. (2005). Blue Ocean Strategy: How to Create Uncontested Market Space and Make Competition Irrelevant. Consulta 15 de enero 2012, de http://www.upacifico.edu.ec/Archivos/Estrategia_oceano_azul.pdf.

Cobo, A. (2001) El neuromarketing, parte principal de las estrategias de marketing. Consulta 26 de noviembre 2011, de http://www. alejandrocobo.com/index.php/neuromarketing.

Anónimo. (2011). Artículos de la sociedad de la información. Consulta 26 de noviembre 2011, de. http://sociedadinformacion.fundacion.telefonica.com/seccion=1188\&idioma=es_ES\&id=2011011216090001\&activo=4.do

Jiménez, C. (1999). Neuromarketing. Consulta 12 de Diciembre de 2011, de http://www.neuromarketing.com.co/.

Malfitano, O., Arteaga, R., y Romano, S. (2007). Neuromarketing, cerebrando negocios y servicios. Buenos Aires. Granica.

Monge, S. (2009) Neuromarca, “El blog sobre neuromarketing en español”. Consulta 13 de Diciembre 2011, de http://neuromarca.com/

Olamendi G. (2001). Neuromarketing. Consulta 7 enero de 2012. De http://www.estoesmarketing.com/Marketing/Neuromarketing.pdf 
